\title{
Azithromycin exposure induces transient microbial composition shifts and decreases the airway microbiota resilience from PM2.5 stress in healthy adults: a randomized, double-blind, placebo- controlled trial
}

Sisi Du

China-Japan Friendship Hospital

Xiaohui Zou

China-Japan Friendship Hospital

Xiaoyan Deng

China-Japan Friendship Hospital

Lianhan Shang

China-Japan Friendship Hospital

Aihua Sun

China-Japan Friendship Hospital

Shengrui Mu

China-Japan Friendship Hospital

Jiankang Zhao

China-Japan Friendship Hospital

Yimin Wang

China-Japan Friendship Hospital

\section{Xiaoxuan Feng}

China-Japan Friendship Hospital

Binbin Li

China-Japan Friendship Hospital

Chunlei Wang

China-Japan Friendship Hospital

Shuai Liu

China-Japan Friendship Hospital

Binghuai Lu

China-Japan Friendship Hospital

Yingmei Liu

China-Japan Friendship Hospital 


\section{Rongrong Zhang}

China-Japan Friendship Hospital

\section{Yigang Tong}

China-Japan Friendship Hospital

\section{Chen Wang}

China-Japan Friendship Hospital

Bin Cao ( $\nabla$ caobin_ben@163.com )

China-Japan Friendship Hospital https://orcid.org/0000-0001-6991-0350

\section{Research}

Keywords: Azithromycin, airway microbiota, healthy volunteers, biodiversity, stability, PM2.5, network

Posted Date: August 18th, 2020

DOI: https://doi.org/10.21203/rs.3.rs-58643/v1

License: (c) (1) This work is licensed under a Creative Commons Attribution 4.0 International License. Read Full License 


\section{Abstract}

Background: Azithromycin, widely used in recent years, can change the airway microbiota in patients with chronic lung diseases. Little data exists regarding the effects of azithromycin administration on airway microbiota among healthy adults. Therefore, we conducted a randomized, double-blind, placebocontrolled trial to assess the process of variation and re-establishment of the airway microbiota after azithromycin exposure in healthy adults.

Methods: Forty-eight healthy volunteers were enrolled and randomly assigned into two groups. $500 \mathrm{mg}$ azithromycin or placebo was administered once daily for 3 days. We collected the induced sputum at the day before the drugs administration (D0), the day after the treatment course was completed (D4), 14 days, 30 days and 60 days post-dosing. 16S rRNA gene sequencing and quantification were applied to the induced sputum samples. We collected the environmental information including air quality data [particulate matter (PM 2.5) and PM 10 , air quality index (AQI) values] that might have an influence on the airway microbiota during the study. The subjects' respiratory tract infection (RTI) events during sampling were recorded.

Results: Azithromycin didn't alter bacterial load but significantly reduced species richness and Shannon index. Azithromycin exposure resulted in decrease in the detection rate and relative abundance of different families belonging to Veillonellaceae, Pasteurellaceae, Leptotrichiaceae, Neisseriaceae and Fusobacteriaceae. By contrast, the relative abundance of taxa belonging to Streptococcus increased immediately after azithromycin intervention. The shifts in the microbial community composition require about 14 days to recover while alpha-diversity recovered later. The high concentration of PM 2.5 contributed to a novel variability in microbial community composition of azithromycin group at D30 (30 days after baseline). The network analysis found that azithromycin altered the microbial interactions within airway microbiota. The influence was still obvious at D14 when the relative abundance of most taxa had returned to the baseline level.

Conclusions: Azithromycin has transient effect in airway microbiota of healthy adults and decreases the ability of the airway microbiota resilient from PM 2.5 stress. The influence of azithromycin on microbial interactions is noteworthy though the airway microbiota has returned to the near-baseline level.

Registration: Chinese Clinical Trial Registry (ChiCTR1800018494), September, 2018. http://www.chictr.org.cn/edit.aspx?pid=31269\&htm=4

\section{Background}

Human airway microbiota forms a complex, balanced ecosystem and serves as the gatekeeper to respiratory health[1]. In both animal models and human studies, it has been shown that the respiratory microbiota is related to the maturation of alveolar architecture and shapes the local immune system[2-5]. A longitudinal study proves that, among the healthy children, the early-life healthy airway microbiota development enforces the defense against respiratory tract infections[6]. Besides, the respiratory 
microbiota may have a role in dealing with the inhaled pollutants[7]. The dysbiosis of airway microbiota, driven by decreased microbial diversity and overgrowth of certain bacterium, may play a role in the occurrence and development of respiratory diseases[8, 9].

Antibiotic use is considered to be one of the most important perturbations for the airway microbiota. In recent years, macrolides have been widely used in infectious diseases and a range of chronic inflammatory airway disorders, including cystic fibrosis, bronchiectasis, chronic obstructive pulmonary disease and persistent uncontrolled asthma[10-14]. Previous randomized placebo-controlled trials have shown that, after exposure to long-term macrolide therapy, a significant reduction in the diversity of respiratory microbiota and Haemophilus influenzaeload can be observed in patients with lung diseases[15, 16]. Although previous analyses well established that macrolide exposure could change the respiratory microbiota in patients with lung diseases, little data exist regarding the variation in airway microbiota after azithromycin (a member of the second generation of macrolides) administration among healthy adults, as well as the longitudinal description about the recovery trajectory of microbiota shifts after the antibiotic perturbation.

Given that the respiratory tract is open to airflow, the airway microbiota may be influenced by the environmental factors. Recent studies in healthy murine show that lung communities clustered significantly by various environmental sources (vendors, cages and shipments)[3]. The exposure to elevated levels of $\mathrm{PM}_{2.5}$ contributes to the change in sputum and pharyngeal microbiota composition, even in the healthy cohort $[17,18]$.

In this longitudinal study, we focus on the airway microbiota dynamic variation in the healthy adults. The 16S rRNA gene sequencing was applied to the induced sputum samples collected before administration of the drugs (azithromycin and placebo) and at four timepoints over 60 days post-dosing. We aim to track the process of variation and re-establishment of biodiversity, microbial interactions and community composition of the airway microbiota after a 3-day azithromycin administration. We also estimate the potential influence of environmental factors (e.g. temperature, the concentration of $\mathrm{PM}_{2.5}$ and $\mathrm{PM}_{10}$ ) on the airway microbiota during the follow-up.

\section{Results}

\section{Baseline characteristics}

The recruitment of the study began on October 8, 2018 and lasted for 2 weeks. We enrolled 48 healthy volunteers with an average age of 26.6 years old (Table 1). The follow-up visits took place from October 2018 to December 2018 (Fig. 1). In total, 221 induced sputum samples were collected (Table 2). All the healthy volunteers resided in Beijing, China and never left Beijing during the study period. Thirty-eight subjects (79.2\%) completed the five timepoints. One subject was diagnosed with acute laryngitis after timepoint D0. Six subjects had common cold at timepoint D30, and three at D60. All dropouts were lost to follow-up right after the last visit. The induced sputum samples were analyzed by sequencing of the $16 \mathrm{~S}$ 
rRNA genes, yielding 24,027,321 reads after quality filtering with $108,720 \pm 107,741(12,525 \sim 719,237)$ reads per sample on average. Thirty-six negative control samples were sequenced alongside the sputum samples obtaining 4,149 $\pm 6,288$ reads per sample. 
Table 1

Demographics of healthy volunteers.

\begin{tabular}{|c|c|c|c|}
\hline Parameter & $\begin{array}{l}\text { Azithromycin Group } \\
(n=24)\end{array}$ & $\begin{array}{l}\text { Placebo } \\
\text { Group } \\
(n=24)\end{array}$ & P Value \\
\hline Male sex, n (\%) & $12(50)$ & $12(50)$ & \\
\hline Age, yr, median \pm IQR & $25 \pm 3.00$ & $25 \pm 3.25$ & $0.60^{d}$ \\
\hline Height, $\mathrm{cm}$, mean $\pm S D$ & $168.75 \pm 7.57$ & $167.21 \pm 8.52$ & $0.51^{\mathrm{e}}$ \\
\hline Weight, kg, mean $\pm S D$ & $62.71 \pm 12.27$ & $61.27 \pm 9.55$ & $0.65^{\mathrm{e}}$ \\
\hline \multicolumn{4}{|l|}{ Race, n (\%) } \\
\hline Han & 23(95.83) & $22(91.67)$ & $1^{f}$ \\
\hline Others & $1(4.17)$ & $2(8.33)$ & \\
\hline \multicolumn{4}{|l|}{ Residence $^{\mathrm{a}}, \mathrm{n}(\%)$} \\
\hline Chaoyang District & $20(83.33)$ & 19(79.17) & $1^{f}$ \\
\hline Others & $4(16.67)$ & $5(20.83)$ & \\
\hline \multicolumn{4}{|l|}{ Occupation, n (\%) } \\
\hline Students & $15(62.5)$ & 19(79.17) & $0.20^{\mathrm{h}}$ \\
\hline Others $^{b}$ & $9(37.5)$ & $5(20.83)$ & \\
\hline \multicolumn{4}{|l|}{ Microbial diversityc } \\
\hline \multicolumn{4}{|l|}{ a-Diversity, mean $\pm S D$} \\
\hline Richness & $414.42 \pm 89.63$ & $408.5 \pm 88.13$ & $0.91^{\mathrm{e}}$ \\
\hline Shannon & $5.99 \pm 0.43$ & $6.00 \pm 0.32$ & $0.80^{\mathrm{e}}$ \\
\hline \multicolumn{4}{|l|}{$\beta$-Diversity } \\
\hline Unweighted Unifrac distance & NA & NA & $0.75^{\mathrm{i}}$ \\
\hline Weighted Unifrac distance & NA & NA & $0.75^{\mathrm{i}}$ \\
\hline
\end{tabular}

a: All the participants were resided in Beijing, China, most of them lived in Chaoyang District, others in Changping District, Shijingshan District, Haidian District, Dongcheng District and Fengtai District. 
b: Other occupations included physicians, company employees, and civil servants.

c: Diversity of the baseline sputum microbiota of the healthy volunteers using samples collected before drug administration.

d: Compared by two-sided Wilcoxon rank sum test.

e: Compared by two-sided Student's t-test.

f: Compared by two-sided Chi-squared test with continuity correction.

h: Compared by two-sided Pearson's Chi-squared test.

i: Compared by PERMANOVA.

IQR is short for interquartile range.

SD is short for standard deviation.

Table 2

Data of sputum samples collected at different timepoints ${ }^{a}$.

\begin{tabular}{|lll|}
\hline Timepoints & $\begin{array}{l}\text { Azithromycin Group } \\
(\mathbf{n}=114)\end{array}$ & $\begin{array}{l}\text { Placebo Group } \\
(\mathbf{n}=107)\end{array}$ \\
\hline D0 & 24 & 24 \\
\hline D4 & 24 & 23 \\
\hline D14 & 24 & 23 \\
\hline D30 & 21 & 20 \\
\hline D60 & 21 & 17 \\
\hline
\end{tabular}

a: The sputum samples were collected before the day of administration of the drugs (D0), the day after the treatment course was completed (D4), 14 days (D14), 30 days (D30) and 60 days (D60) post-dosing. D: day

b: Thirty-eight of the all subjects completed the five timepoints. Six subjects had a cold at timepoint D30. Three subjects had common cold at timepoint D60. One subject was diagnosed with acute laryngitis after timepoint D0. All dropouts were lost to follow-up right after the last visit. 


\section{Minimal influence of procedural contaminations on sputum samples sequencing}

It was important to identify the impact of contamination on sequencing results, because the consumables and reagents used in the experiments, such as DTT, DNA isolation and library preparation might contain bacterial DNA. The microbial community composition between sputum samples and control samples was quite distinct based on both unweighted UniFrac and weighted UniFrac distance (PERMANOVA, $R^{2}=0.38,0.28 ; P=0.001,0.001$ Additional file 1: Figure $S 1 a-b$ ). The five Zero-radius Operational Taxonomic Units (ZOTUs, $100 \%$ sequence similarity) detected in sputum samples with the highest abundance comprised $23.9 \%$ of all sequences, while comprising $9.8 \%$ of all sequences detected in control samples (Additional file 1: Figure S1c). The most abundant ZOTU detected in control specimens (ZOTU36) accounted for only $0.029 \%$ of all sequences detected in sputum specimens (Additional file 1: Figure S1d). We performed microbial ecology analysis using the decontam package whose function was to identify contaminants in sequencing data based on statistical approach[19]. We used both the frequency and prevalence methods with threshold 0.1 and 0.5 respectively and confirmed only 3 contaminants ZOTUs (ZOTU1714, ZOTU2282, ZOTU2462) in our data (Additional file 1: Figure S1e-f, Additional file 2: Table S1). The relative abundance of the 3 ZOTUs were very low in both sputum and negative control samples (comprising $1.96 \times 10^{-4} \%, 6.24 \times 10^{-5} \%, 4.58 \times 10^{-5} \%$ of all sputum samples sequences and $1.34 \times 10^{-3} \%, 1.87 \times 10^{-2} \%, 1.34 \times 10^{-2} \%$ of all negative control samples sequences). ZOTU1714, ZOTU2282 and ZOTU2462 only appeared in $2.26 \%, 3.62 \%$ and $1.81 \%$ of sputum samples and $2.78 \%, 8.33 \%$ and $2.78 \%$ of control samples, respectively. The rare and low-prevalence sequences often had no effect on the microbial diversity[20]. In conclusion, we found few evidences of procedural contaminations influencing the species detected in sputum. After removal of the 3 ZOTUs, a total of 2827 ZOTUs were analyzed.

\section{Stable bacterial DNA burden in healthy volunteers' airways after azithromycin administration}

We quantified the bacterial DNA of every sputum sample using droplet digital

PCR system. There was considerable variation across individual sputum samples (the first quartile and the third quartile, $1.8 \times 10^{7} \sim 9.7 \times 10^{7}$ copies $/ g$ ). However, we found no significant difference in sputum bacterial DNA burden across different timepoints in either azithromycin or placebo group (Wilcoxon signed-rank test, Bonferroni adjusted $P$ values (q) $>0.05$ for all Fig. 2). 


\section{The shifts in sputum phylogenetic diversity after antibiotic exposure}

The baseline sputum microbiota of healthy volunteers (D0) showed no significant difference between the two groups (two-sided Student's t-test and PERMANOVA, $P>0.05$ for all Table 1). Immediately after the azithromycin course was completed (D4), both the richness and Shannon index were dramatically reduced compared to D0 (two-sided paired t-test, $q=9.799 \times 10^{-6}$ and $q=3.92 \times 10^{-6}$, respectively Fig. 3a-b). By D14, the species richness still remained decreased, but Shannon index significantly increased compared to $D 4$ (two-sided paired t-test, $q=1, q=1.062 \times 10^{-3} \mathrm{Fig}$. 3a-b), suggesting that the relative abundance of surviving microorganisms affected by antibiotic started to recover. During the two-month follow-up, the species richness significantly increased at D30 compared to D14 (two-sided paired t-test, $\mathrm{q}=4.366 \times 10^{-4}$ ) and completely recovered at D60 compared to D0 (two-sided paired t-test, $q=0.387$ Fig. 3a). Meanwhile, we found no significant differences in richness and Shannon index among the healthy volunteers in the placebo group across the five timepoints. (Additional file 3: Figure S2a-b).

Although pairwise PERMANOVA testing using unweighted UniFrac distance showed significant compositional differences between timepoint D0 and timepoints D4, D14, D30 and D60 $\left(R^{2}=0.083,0.105\right.$, $0.081,0.054 ; q=0.01,0.01,0.01,0.05$ ), principal coordinate analysis (PCoA) demonstrated that the sputum microbial compositions gradually returned towards their initial composition after profound differences at D4 (Fig. 3c). The microbial profiles recovery was also reflected by a highest unweighted UniFrac distance observed at D4, along with significantly descending magnitude over time compared to the placebo group (Fig. 3d). When we used weighted UniFrac distance accounting for relative abundance to perform pairwise PERMANOVA testing in azithromycin group, the significant change in microbial community composition compared to D0 was identified at D4, but not D14 $\left(R^{2}=0.104,0.036 ; q=0.01,0.92\right.$ Fig. 3e).

\section{Microbial taxonomic variation during 60 days' follow-up}

We investigated the microbial taxonomic variation across the five timepoints by comparing the detection rate and relative abundance of the ZOTUs. We involved 294 ZOTUs whose relative abundance was greater than $0.01 \%$ and detection rate was greater than $50 \%$ at baseline in the analysis. After exposing to azithromycin, the detection rate of 112 ZOTUs decreased at D4. There was a depletion of some families, such as Veillonellaceae (17.86\%), Leptotrichiaceae (9.82\%), Fusobacteriaceae (8.93\%), Neisseriaceae (7.14\%), Actinomycetaceae (7.14\%), Pasteurellaceae (6.25\%), Prevotellaceae (6.25\%) and Lachnospiraceae (5.36\% Figure 4, Additional file 4: Table S2). By D14, 74 (66.07\%) of the ZOTUs whose 
detection rate decreased significantly between D0 and D4 exhibited significant differences. By contrast, only $34(30.36 \%)$ of the above mentioned ZOTUs showed differences in their relative abundance, suggesting that the loss of species usually recovered later than the abundance of them (Figure 4, Additional file 4: Table S2). Most of the ZOTUs returned to D0 level during the follow-up, but several ZOTUs, such as Fusobacteriaceae ZOTUs, Pasteurellaceae ZOTUs, Neisseria ZOTU and Leptotrichiaceae ZOTUs remained low in detection rate at D30, and had not been back to the initial state by the end of observational period (Fig. 4, Additional file 4: Table S2). We observed an enrichment of the family Streptococcaceae ZOTUs in the relative abundance at D4, immediately post-administration. Most of them rapidly decreased and returned to the baseline level at D14 (Fig. 4, Additional file 4: Table S2).

Among 294 ZOTUs, the detection rate of 204 ZOTUs returned to the D0 level at D14 and only $2.5 \%$ of them exhibited significant differences compared to D0 again at D30 (Additional file 5: Figure S3a, Additional file 6: Table S3). The relative abundance of 227 ZOTUs was of no significant difference between D0 and D14 while $9.7 \%$ of those ZOTUs had novel variation in the relative abundance at D30 (Additional file 5: Figure S3b-c, Additional file 6: Table S3). At family and genus levels, the relative abundance of $72.9 \%(n=35)$ of families and $79.8 \%(n=71)$ of genera had been not significantly different compared to baseline at D14 while 7 families and 10 genera had a shift at D30 again (Additional file 5: Figure S3d).

We found no significant microbial variation in the detection rate and relative abundance in the placebo group across the five timepoints (Additional file 7: Figure S4).

\section{Attenuated resilience in response to environmental factors disturbance}

Higher biodiversity richness was observed in samples collected at D30 but there was no significant difference in Shannon index between D14 and D30 (two-sided paired t-test, q=1 Fig. 3b). Besides, a significantly compositional shift was also observed (pairwise PERMANOVA, $R^{2}=0.081, q=0.01$ Fig. $3 e$ ), in line with an increased weighted UniFrac distance $(0.287 \pm 0.068$ Fig. $3 f)$ at this timepoint, suggesting that some variation occurred in the abundance of sputum taxa and prevented the recovery. We found that the daily mean concentrations of $\mathrm{PM}_{2.5}, \mathrm{PM}_{10}$, $\mathrm{AQI}$ values, daily mean humidity and temperature were significantly different (Kruskal-Wallis rank sum test, $P<0.05$ Additional file 8: Figure S5) among the five periods. The distance-based redundancy analysis (db-RDA) was performed to explain the environmental stresses on the sputum microbiota variation. By D4, not only the antibiotic, but also humidity had a significant influence on the structure of the microbiota $\left(R^{2}=0.027, q=0.004 ; R^{2}=0.052, q=0.012\right.$ Fig. 5a). After the variance decomposition, we found that the antibiotic could individually explain $2.7 \%$ of microbiota variance $(P=0.001)$ and humidity could individually explain $2.2 \%$ of microbiota variance $(P=0.001)$. Hence, we concluded that administration of azithromycin was related to sputum microbiota, 
but the environmental factor, humidity, might also shape the microbial communities. By D30, db-RDA showed that the high concentration of $\mathrm{PM}_{2.5}$ significantly contributed to the variability in microbiota composition, but not antibiotics $\left(R^{2}=0.026,0.013, q=0.004 ; q=0.088\right.$ Fig. $\left.5 b\right)$, suggesting environmental factors served as a novel shock to disturb the sputum microbiota which had recovered to the nearbaseline microbial community composition.

In placebo group, the environmental factors weren't associated with the sputum microbiota in healthy volunteers (Additional file 9: Figure S6).

\section{The effects of azithromycin on airway microbial interactions within the microbial community network}

To explore the changes of microbial interaction after exposure to azithromycin, a bacterial community network analysis was performed. The azithromycin group networks became less complicated and stabilized after the antibiotic exposure regarding decreases in the total number of vertices, edges, connectance, average degree, average clustering coefficient and centralization closeness (Additional file 10: Figure S7). The co-occurrence patterns of microbial communities before and after azithromycin administration were quite distinct. We identified the importance of ZOTUs using closeness centralization scores and degree centralization scores. ZOTUs belonging to families Fusobacteriaceae and Porphyromonadaceae set a central position at D0 and those assigned to families Prevotellaceae, Lachnospiraceae and Veillonellaceae played important roles as well. After exposure to antibiotics, families Veillonellaceae and Leptotrichiaceae came to the first while families Fusobacteriaceae and Lachnospiraceae's superiority in the network declined. By D60, families Fusobacteriaceae and Lachnospiraceae returned to their original position again (Fig. 6a). Along with the decrease in detection rate or relative abundance of the major species after administration of antibiotics, the synergistic effect in the co-occurrence networks occupied the main status (Fig. 6a). Despite having a few overlapped edges between timepoint $\mathrm{D} 0$ and other timepoints networks, the closeness of shared nodes was quite different. Though, by D14, the relative abundance of most ZOTUs showed no significant difference compared to D0, the closeness of them still remained decreased (Fig. 6b-C). In control group, families Fusobacteriaceae, Lachnospiraceae, Prevotellaceae and Veillonellaceae kept occupying the important positions within the framework along with time (Additional file 11: Figure S8a). The co-occurrence patterns of the microbial communities were quite similar, showing that they contained more overlapped edges and the closeness of shared nodes exhibited very small differences among the five timepoints networks (Additional file 11: Figure S8b-c). It was interesting to see that both positive and negative correlation existed in the networks, suggesting that the interactions among the species made the bacterial community stable. 


\section{The effects of azithromycin on oral cavity microbiota}

The bacterial DNA burden in oral wash samples (the first quartile and the third quartile, $1.1 \times 10^{6} \sim 4.5 \mathrm{x}$ $10^{6}$ copies/g) was significantly lower than sputum samples (Wilcoxon signed-rank test, $\mathrm{q}<0.05$ for all Additional file 12: Figure S9). Oral cavity microbiota had lower species richness at baseline compare to sputum microbiota ( $484.33 \pm 89.57$ vs $414.42 \pm 89.63$ two-sided paired t-test, $q=0.0033$ Additional file 13 : Figure S10, Additional file 14: Figure S11). PERMANOVA testing using both unweighted UniFrac distance and weighted UniFrac distance found that the microbial community composition at D0 between the two niches was quite distinct $\left(R^{2}=0.04,0.05 ; P=0.029,0.033\right.$ Additional file 14: Figure S11). Azithromycin altered the alpha-diversity and the community composition of the oral microbiota (Additional file 14: Figure S11). However, compared to the sputum microbiota, oral cavity microbiota had a different pattern of change over time (Additional file 14: Figure S11). Bacterial community network analysis showed that the interactions among the species in oral cavity microbiota community recovered earlier than sputum microbiota (Additional file 15: Figure S12, Additional file 16: Figure S13). Detailed explanation can be seen in the supplementary information (Additional file 17: Figure S14, Additional file 18: Figure S15, Additional file 19: Figure S16, Additional file 20: Figure S17, Additional file 21: Supplementary text).

\section{Discussion}

This is the first randomized, double-blind, placebo-controlled trial that investigates the microbial shifts in airways after exposure to azithromycin among heathy adults. The core findings of our study are that airway microbiota of healthy volunteers show resilience and abilities for recovery from azithromycin perturbation. There is a depletion of families Veillonellaceae, Pasteurellaceae, Leptotrichiaceae, Neisseriaceae and Fusobacteriaceae while an enrichment of family Streptococcaceae immediately after azithromycin administration. The shifts in the microbial community composition require about 14 days to recover while alpha-diversity recovers later. Azithromycin exposure alters the microbial interactions within airway microbial community networks and decreases the ability of the airway microbiota resilient from $\mathrm{PM}_{2.5}$ stress. The influence of azithromycin on microbial interactions is noteworthy though the relative abundance of most taxa has returned to the baseline level.

In our study, we find that exposure to azithromycin resulted in decrease in the detection rate and relative abundance of different families belonging to Veillonellaceae, Pasteurellaceae, Leptotrichiaceae, Neisseriaceae, which are sensitive to azithromycin[21]. Those taxa may act as a major ecological change causing the decrease in alpha-diversity immediately after antibiotic administration. The finding is similar to the previous researches that macrolides have a selective impact on $\mathrm{H}$. influenzae or species assigned to Pasteurellaceae within airway microbiota both in asthma and non-cystic fibrosis bronchiectasis patients and cause significantly reduced diversity after antibiotic exposure[15, 16]. By contrast, the relative abundance of taxa belonging to Streptococcus increases immediately after azithromycin administration. Among the asthma patients, the azithromycin administration is correlated with an increase in cultured azithromycin-resistant Viridans streptococci, in line with the increased abundance of 
Streptococcus reported here[15]. The macrolide resistance in Streptococcus spp is increasing around the world in recent years[22-24]. In China, more than $90 \%$ of the Streptococcus pneumoniae isolates are resistant to macrolide both in children and adults[25,26]. The high antibiotic-resistance may provide those species with an advantage to effectively colonize the airway after administration. Fortunately, the upgradations of Streptococcus species are transient and return to the D0 level after antibiotic ceases in our study. Our results suggest that the effects of azithromycin on airway microbiota composition are modest. The microbial biodiversity in our study requires about 14-60 days to recover, while it takes 1.5 months for gut microbiota after antibiotic disturbance[27]. Besides, azithromycin plays few roles in altering the total bacterial load in the airways.

$\mathrm{PM}_{2.5}$ exposure is associated with increased all-cause and respiratory mortality, impaired lung function, exacerbations of chronic lung diseases, lung cancer, even Acute Respiratory Distress Syndrome (ARDS) [28-34]. Some studies have found that $\mathrm{PM}_{2.5}$ exposure could alter human pharyngeal[18], airways[17] and gut microbiota composition[35] because atmospheric particulate matter commonly carries microbes rich in Firmicutes, Proteobacteria and Bacteroidetes phyla[36]. During our study period, the concentration of $\mathrm{PM}_{2.5}$ is very high in the week around timepoint D30. However, we do not observe the variation in airway microbiota community composition of placebo group subjects. One of the reasons may be that all the subjects work indoor for most of time and the duration of daily pollutant exposure is short. What's more, the microbiota communities can show remarkable stability against perturbations[37] and the respiratory microbiota normally has the ability to deal with the inhaled pollutants[7]. The microbiota stability may comprise two concepts. One is resistance, meaning that the composition and function of a given microbiota stand unchanged in response to a disturbance and the other is resilience, which describes that the microbiota is able to return to the predisturbed state[38]. On the contrary, the airway microbiota of azithromycin group subjects, an almost re-established microbial ecology after exposure to antibiotics, cannot bear the same burden of $\mathrm{PM}_{2.5}$ pollution and has a novel shift in microbial community composition at D30. From the previous studies, we find that the individuals with dysregulated respiratory tract condition, such as respiratory illness and smoker, or disequilibrated airway ecology, are particularly susceptible to the environmental stress or disturbance[39, 40]. Therefore, we assume that, in our study, the stability of airway microbiota decreases because of azithromycin exposure, which results in attenuated resilience in response to environmental factors.

We perform network analysis to support above-mentioned assumption. The functional stability of microbial communities depends on the complicated microbe-microbe interactions[41]. Studies about soil microbiota confirm that the network complexity is consistent with the ability to cope with the diverse environmental changes[42]. After exposure to azithromycin, the airway microbiota networks in our study become simple, indicating the attenuated resilience. The combination of both mutualistic and antagonistic correlations embraced in the networks is important to keep ecological equilibrium[43]. The microorganisms within the communities can cooperate and compete for nutrition to prevent the growth or decline of certain one[37]. In our results, the weakening antagonistic effects due to losing some species contribute to the novel variation in airway microbiota when the environment factors change. It is reported 
that the mode of action of keystone taxa which mediates multiple interactions within networks is associated with the stability of the microbial communities[44]. Our study finds that antibiotic perturbation targets the crucial taxa by altering the position of them in the framework, which may, in turn, precipitate the microbiota structure fragility. Moreover, the influence of azithromycin on microbial interactions within airway microbial communities is noteworthy though the relative abundance of most taxa has returned to the baseline level.

Our study has following limitations. Firstly, we use induced sputum to investigate the variation in airway microbiota after antibiotic intervention. The induced sputum is a variable mixture that may contain materials from human airways and oral cavity. We ask the subjects to fast for 4-6 hours and clean oral cavity. To determine the contribution of each source, oral washes were also performed by having the subjects gargle with $10 \mathrm{ml}$ sterile $0.9 \%$ saline for 60 seconds immediately before sputum induction. We found that the bacterial DNA burden in oral wash samples was significantly lower than sputum samples. Oral cavity microbiota had lower species richness and a significantly different microbial community composition compared to sputum microbiota. Besides, compared to the sputum microbiota, oral cavity microbiota had a different change pattern and recovered earlier after antibiotics exposure. However, for successful 16S rRNA gene library preparation, we increased the volume of oral wash samples, up to $3 \mathrm{ml}$, for DNA extraction. It wasn't consistent with the volume, 750ul, of the induced sputum for using, which might decrease the comparability of the sequencing data. Secondly, human airway microbiome may be influenced by numerous factors. To avoid deviations as much as possible, we strictly execute a randomized, double-blind, placebo-controlled pattern across the entire study and experimentation process. The stability in airway microbiota of placebo group subjects gives us evidence to believe our conclusions drawn from that of azithromycin group. At last, we only follow up the volunteers for 60 days, the longer-term effects after azithromycin administration demand more attention in future.

\section{Conclusions}

In summary, we conclude that azithromycin exposure has transient effect in airway microbial community composition of healthy adults. Azithromycin alters the microbial interactions within airway microbial community networks and decreases the airway microbiota resilience from $\mathrm{PM}_{2.5}$ stress. The influence of azithromycin on microbial interactions is noteworthy though the relative abundance of most taxa has returned to the baseline level.

\section{Methods}

\section{Study design and population}

We designed a randomized, double-blind, placebo-controlled trial to explore the influence of azithromycin on the airway microbiota of healthy adults. Inclusion criteria for the healthy volunteers were men and women aged over 18 years old and had no history of smoking. Detailed inclusion and exclusion criteria are provided in (Additional file 22: Table S4). Finally, forty-eight healthy volunteers were enrolled into this 
study and stratified by gender and randomly assigned to either the azithromycin or the placebo group (1:1) using a computer-generated random number table. $500 \mathrm{mg}$ azithromycin or $500 \mathrm{mg}$ starch tablets were administered once daily for 3 days. The drugs were contained in an identical opaque white capsule respectively and packed in sealed kraft paper bags. All healthy volunteers, investigators and study research staff were masked to intervention allocation.

\section{Sputum collection}

The healthy volunteers were asked to fast for 4-6 hours and clean oral cavity, including brushing teeth and gargling with $10 \mathrm{ml} 0.9 \%$ saline for 60 seconds at our laboratory immediately before the sputum induction. The oral wash samples were collected. Sterile saline was used as an oral wash control. And then, they were subjected to inhale $4.5 \%$ sterile hypertonic saline nebulized from an inhalation delivery (PARI, Germany) for 10 minutes and endeavored to cough throughout the process. Most subjects could expectorate an adequate sample (sputum $\geq 1 \mathrm{ml}$ with as little saliva as possible) after the first inhalation. For those who failed to meet the demands, we extended the duration of aerosol inhalation cumulatively up to 60 minutes with pause every 10 minutes or allowed them to cough for the second time later. Sputum was collected in sterile containers, weighed and $2 \mathrm{ml}$ of $0.1 \%$ dithiothreitol (DTT) (Sigma-Aldrich, Poole, UK) in phosphate-buffered saline (PBS) was added for liquefaction. The $0.1 \%$ DTT-PBS in sputum collection cups and sterile saline in the atomization device served as negative control samples. After incubated at room temperature for 15 minutes on a rolling mixer, all the samples were stored in sterile tubes at $-80^{\circ} \mathrm{C}$ immediately until processed. The sputum samples were collected at the day before the drugs administration (D0), the day after the treatment course was completed (D4), 14 days, 30 days and 60 days post-dosing. We asked the participants to maintain a consistent time point in induced sputum collecting within individual longitudinal samples timepoint.

\section{Environmental and volunteers' follow-up information collection}

We collected the environmental information that might have an influence on the airway microbiota, including air pollution data, humidity, temperature and history of leaving Beijing city during the study period. We obtained the daily mean concentration of $\mathrm{PM}_{2.5}$ and $\mathrm{PM}_{10}$, air quality index (AQI) values, daily mean humidity and temperature in Beijing city from China air quality online monitoring platform (The data is available from $h t t p s: / / w w w$.aqistudy.cn/). Relevant information recorded before every sampling included whether the subjects developed respiratory tract infection symptoms and history of antibiotic administration. 


\section{DNA extraction and 16S rRNA gene sequencing}

DNA extraction from sputum and oral wash samples, paralleled with control samples, was performed using the Maxwell ${ }^{\circledR}$ RSC Whole Blood DNA Kit (Promega, USA). 750 ul sample was transferred to the eppendorf tube and centrifuged for 5 minutes at $14,000 \mathrm{~g}$. The supernatant was discarded and precipitation was re-suspended in PBS to 720 ul with 80 ul proteinase $\mathrm{K}$ (Tiangen, China) added into. Then, we transferred re-suspended material to a lysing matrix A tube (Qbiogene, Carlsbad, CA). Cells were lysed by bead beating using a FastPrep system (Qbiogene, Carlsbad, CA) for 3 cycles of 30 seconds at $6.0 \mathrm{~m} / \mathrm{sec}$ and the mixture was subsequently incubated at $56^{\circ} \mathrm{C}$ for 1 hour. After centrifugation for 5 minutes at 14,000 rpm, $500 \mathrm{ul}$ supernatant was then transferred to the $1 \#$ well of the cartridge. $70 \mathrm{ul}$ of Elution Buffer was added to the bottom of each elution tube. Extracted DNA was quantified using a Qubit 3.0 Fluorometer (Thermo Fisher Scientific, Waltham, MA, United States) and stored at $-20{ }^{\circ} \mathrm{C}$

The V3-V4 hypervariable region of the 16S rRNA gene of all samples was amplified by PCR according to the 16S Metagenomic Sequencing Library Preparation guide provided by Illumina technology[47]. The amplicon primers were Forward Primer $=5^{\prime}$

TCGTCGGCAGCGTCAGATGTGTATAAGAGACAGCCTACGGGNGGCWGCAG and Reverse Primer=5' GTCTCGTGGGCTCGGAGATGTGTATAAGAGACAGGACTACHVGGGTATCTAATCC. The PCR conditions were $95^{\circ} \mathrm{C}$ for $3 \mathrm{~min}$; followed by 25 cycles of $95^{\circ} \mathrm{C}$ for $30 \mathrm{~s}, 56^{\circ} \mathrm{C}$ for $30 \mathrm{~s}$, and $72^{\circ} \mathrm{C}$ for $30 \mathrm{~s}$; and then $72^{\circ} \mathrm{C}$ for $5 \mathrm{~min}$. The amplicons were performed in $25 \mu \mathrm{L}$ reactions with $12.5 \mathrm{ng}$ template DNA. Index PCR was the 2nd stage PCR step that attached dual indices and Illumina sequencing adapters using the Nextera XT Index Kit, Index Primer 1(N7xx) and Index Primer 2(S5xx) (Illumina, San Diego, CA) in 50 ul reaction. The PCR conditions were $95^{\circ} \mathrm{C}$ for $3 \mathrm{~min}, 8$ cycles at $95^{\circ} \mathrm{C}$ for $30 \mathrm{~s}, 55^{\circ} \mathrm{C}$ for $30 \mathrm{~s}$, and $72^{\circ} \mathrm{C}$ for $30 \mathrm{~s}$, then $72^{\circ} \mathrm{C}$ for $5 \mathrm{~min}$. The resulting amplicons were purified using Agencourt AMPure XP (Beckman Coulter) and quantified using a Qubit 3.0 Fluorometer. The pooled library, including negative controls, was sequenced on an Illumina Miseq platform (Illumina, San Diego, CA, United States) using pair-end sequencing $(2 \times 300 \mathrm{bp})$.

\section{Bacterial DNA quantification}

Bacterial DNA of sputum samples was quantified using a QX200 Droplet Digital PCR System (BioRad, Hercules, CA). Two replicates were used per sample. Primers, cycling conditions and workflow were performed according to a previously published protocol[3]. 


\section{Statistical analysis}

The sequencing data were processed and analyzed using the software VSEARCH version 2.7.1[48], USEARCH version 10.0[49]. The sequencing data were merged (minimum 50 bp overlap), trimmed of primers and indexes, and quality filtered (fastq maxee rate 0.01) with VSEARCH version 2.7.1. Reads were denoised into Zero-radius Operational Taxonomic Units (ZOTUs) with UNOISE3[50] and chimeras were filtered using SILVA version 123 reference database. Taxonomic assignment of ZOTUs was performed by SINTAX[51].

Statistical analysis was performed in R version 3.6.1 via the Rstudio interface. To identify the contaminating DNA coming from experiment operation, the decontam package was used. The feature table covering all samples and the concentration of DNA in each eligible library as measured by fluorescent intensity were input to the function. Species richness, Shannon index, unweighted UniFrac distance and weighted UniFrac distance were computed and ordination analyses were conducted using vegan, ape, ggplot2 and GUniFrac packages with all ZOTUs. Principal coordinates analysis and permutational analysis of variance (PERMANOVA) were performed based on both unweighted UniFrac distance and weighted UniFrac distance. Distance-based redundancy analysis (db-RDA) was performed using weighted UniFrac distance. The diversity comparations between the same group were calculated by paired t-test or Wilcoxon signed-rank test. The diversity comparations between the different groups were calculated by Student's t-test or Wilcoxon rank sum test. We performed linear mixed model and McNemarBowker test to compare the variation in relative abundance and detection rate of the ZOTUs whose relative abundance was greater than $0.01 \%$ and detection rate was greater than $50 \%$ at baseline, respectively. Network analysis was used to explore co-occurrence patterns of ZOTUs within the groups. We involved the ZOTUs whose relative abundance was greater than $0.01 \%$ and detection rate was greater than $50 \%$ at baseline to obtain Spearman rank correlation matrix ( $r \geq 0.6$ and $q \leq 0.05)$. The networks were constructed and visualized using igraph package. $P$ values were adjusted using the Bonferroni-control procedure. The heatmaps were visualized by ComplexHeatmap package[52].

\section{Abbreviations}

RTIs: respiratory tract infections; D0: baseline; D4: four days after baseline; D14: fourteen days after baseline; D30: thirty days after baseline; D60: sixty days after baseline; PM: particulate matter; AQI: air quality index; DTT: dithiothreitol; PBS: phosphate-buffered saline; PERMANOVA: Permutational multivariate analysis of variance; ZOTUs: Zero-radius Operational Taxonomic Units; PCR: polymerase chain reaction; PCoA: principal coordinate analysis; db-RDA: distance-based redundancy analysis.

\section{Declarations}

\section{Ethics approval and consent to participate}


The study was carried out in China-Japan Friendship Hospital, Beijing, China. Ethical approval was obtained from the China-Japan Friendship Hospital Ethics Committee (Approval No. 2018-120-K86). All subjects provided written consents. The study was registered at Chinese Clinical Trial Registry (ChiCTR1800018494).

\section{Consent for publication}

Not applicable

\section{Availability of data and materials}

The sequence data have been deposited in the NCBI Sequence Read Archive under accession PRJNA565553. (https://www.ncbi.nlm.nih.gov/bioproject/PRJNA565553). Feature, taxonomy, metadata tables and a reproducible workflow of the analysis are available for download at https://github.com/Zoey-Du/Sputum_microbiota.

\section{Competing interests}

The authors declare that they have no competing interests.

\section{Funding}

Funding provided by the CAMS Innovation Fund for Medical Sciences (CIFMS 2018-I2M-1-003), the National Science Grant for Distinguished Young Scholars (81425001/H0104), the Fundamental Research Funds for the Central Universities and Research projects on biomedical transformation of China-Japan Friendship Hospital (PYBZ1820) and the Ministry of Science and Technology of China (2017ZX10103004), the National Key R\&D Program of China (2017YFC1309301, 2017YFC1309300).

\section{Authors' contributions}

Conception and design: BC, SSD. Acquisition of data: SSD, XYD, LHS, AHS, SRM, YMW, XXF. Analysis and interpretation of data: SSD, XHZ, JKZ, XYD, SL. Drafting or revising of manuscript: SSD, XHZ, JKZ, XYD, LHS, BBL, CLW. Final approval of manuscript: SSD, LHS, BHL, YML, BC.

\section{Acknowledgements}

The authors thank for Mengxue Li for randomization and masking, the study participants and the staff from the CAP-China network. 


\section{Supplementary Information}

Additional file 1: Figure S1. Procedural contaminations on sputum samples sequencing analysis. (A) PCoA plot based on unweighted UniFrac distance (A) and weighted UniFrac distance (B) between sputum samples and negative control samples. The ellipses represent the $68 \%$ confidence interval for each timepoint. ZOTUs are ranked in descending order of mean relative abundance of sputum samples (C) and control samples (D). Decontam package confirms 3 contaminants ZOTUs (ZOTU1864, ZOTU2014, ZOTU2236) in our data using both the frequency $(E)$ and prevalence $(F)$ methods with threshold 0.1 and 0.5 respectively. * a noncontaminant ZOTU.

Additional file 2: Table S1. Procedural contaminations on sputum and oral wash samples sequencing analysis.

Additional file 3: Figure S2. Airway microbial diversity and community composition in placebo group. (A) Species richness. (B) Shannon index. The boxplots represent the diversity measures for the subjects (centre line, mean; box limits, \pm standard deviation; whisker limits, maximum/minimum). The points are connected across timepoints by grey lines. (C) PCoA plot based on unweighted UniFrac distance. (D) PCoA plot based on weighted UniFrac distance. The ellipses represent the $68 \%$ confidence interval for each timepoint.

Additional file 4: Table S2. The mean relative abundance and detection rate of included ZOTUs.

Additional file 5: Figure S3. Sankey plots to describe the number of ZOTUs whose detection rate $(A)$ and relative abundance $(B)$ shifts or returns to the baseline level across the five timepoints. For the taxa whose relative abundance has been of no significant difference from the D0 level at D14, novel shifts in their relative abundance at timepoint D30 are shown at ZOTU (C), family and genus levels (D). Red represents that there is a significant difference in the relative abundance of the species at D30 compared to D0 level and blue represents that there is a significant difference in the relative abundance of the species at D30 compared to D14.

Additional file 6QTable S3. The number of ZOTUs whose relative abundance or detection rate shifts or returns to the baseline level across the five timepoints.

Additional file 8: Figure S4. Microbial taxonomic variation in placebo group during 60 days' follow-up.

Additional file 8: Figure S5. The daily mean air pollution data and temperature and humidity during the sputum samples collecting. * $\mathrm{AQI}\left(161 \pm 73 \mathrm{ug} / \mathrm{m}^{3}\right)$, the concentration of $\mathrm{PM}_{2.5}\left(90 \pm 36 \mathrm{ug} / \mathrm{m}^{3}\right)$ and $\mathrm{PM}_{10}$ $\left(213 \pm 159 \mathrm{ug} / \mathrm{m}^{3}\right)$ are highest at D30 among the five timepoints (Nemenyi test, $\left.\mathrm{q}<0.05\right)$.

Additional file 9: Figure S6. Distance-based redundancy analysis (db-RDA) based on weighted UniFrac distance in placebo group. (A) The relation of the environmental factors and the sputum microbiota variation at D4. (B) The relation of the environmental factors and the sputum microbiota variation at D30. The ellipses represent the $95 \%$ confidence interval for each timepoint. 
Additional file 10: Figure S7. The topological properties of microbial networks in azithromycin and placebo groups.

Additional file 11: Figure S8. The network analysis in placebo group. (A) Networks of co-occurring ZOTUs in airway microbiota for timepoints D0, D4, D14, D30 and D60. Nodes are colored by ZOTU families, with size proportional to mean relative abundance, and edge width proportional to confidence score. (B) The number of shared edges between network D0 and timepoints D4, D14, D30, D60. (C) The closeness centralization of shared nodes between network D0 and timepoints D4, D14, D30, D60.

Additional file 12: Figure S9. The bacterial DNA burden in oral wash samples and sputum samples.

Additional file 13: Figure S10. Procedural contaminations on oral wash samples sequencing analysis. (A) PCoA plot based on unweighted UniFrac distance (A) and weighted UniFrac distance (B) between oral wash samples and negative control samples. The ellipses represent the $68 \%$ confidence interval for each timepoint. ZOTUs are ranked in descending order of mean relative abundance of oral wash samples (C) and control samples (D). Decontam package confirms 7 contaminants ZOTUs in our data using both the frequency $(E)$ and prevalence $(F)$ methods with threshold 0.1 and 0.5 respectively. * a noncontaminant ZOTU.

Additional file 14: Figure S11. Oral cavity and airway microbial diversity and community composition in azithromycin group across the five timepoints. (A) Species richness. (B) Shannon index. (C) PCoA plot based on unweighted UniFrac distance. (D) PCoA plot based on weighted UniFrac distance. The ellipses represent the $68 \%$ confidence interval for each timepoint.

Additional file 15: Figure S12. The topological properties of microbial networks in oral cavity and airway microbiota.

Additional file 16: Figure S13. The network analysis in oral cavity microbiota in azithromycin group. (A) Networks of co-occurring ZOTUs in oral cavity microbiota for timepoints D0, D4, D14, D30 and D60. Nodes are colored by ZOTU families, with size proportional to mean relative abundance, and edge width proportional to confidence score. (B) The number of shared edges between oral cavity and airway networks at timepoints D0, D4, D14, D30, D60. (C) The closeness centralization of shared nodes between oral cavity and airway networks at timepoints D0, D4, D14, D30, D60.

Additional file 17: Figure S14. The microbial profile of oral wash samples and sputum samples. (A) Clustering of the oral wash sample microbiota at ZOTU level according to the detection rate. (B) Clustering of the oral wash sample microbiota at ZOTU level according to the mean relative abundance. Average relative taxa abundance comparisons between oral cavity and sputum microbiota at the family (C) and genus (D) levels. $\uparrow$ significantly higher in sputum samples; $\downarrow$ significantly higher in oral wash samples.

Additional file 18: Figure S15. Microbial taxonomic variation after azithromycin administration in oral wash samples during 60 days' follow-up. ${ }^{*} P$ value $\geq 0.05$ : there isn't a significant difference in the relative 
abundance of the species at D14 compared to D0; $P$ value< 0.05 : there is a significant difference in the relative abundance of the species at D14 compared to D0.

Additional file 19: Figure S16. Sankey plots to describe the number of ZOTUs whose detection rate (A) and relative abundance (B) shifts or returns to the baseline level across the five timepoints in oral cavity microbiota. The shared ZOTUs whose detection rate (C) and relative abundance (D) had significant difference in oral wash samples (OW) microbiota and induced sputum samples (IS) microbiota.

Additional file 20: Figure S17. Distance-based redundancy analysis (db-RDA) based on weighted UniFrac distance in oral wash samples in azithromycin group. (A) The relation of the environmental factors, antibiotic uses and the oral cavity microbiota variation at D4. (B) The relation of the environmental factors, antibiotic uses and the oral cavity microbiota variation at D30. The ellipses represent the $95 \%$ confidence interval for each timepoint.

Additional file 21: Supplementary text.

Additional file 22: Table S4. The inclusion and exclusion criteria for the subjects

\section{References}

1. Man WH, de Steenhuijsen Piters WAA, Bogaert D: The microbiota of the respiratory tract: gatekeeper to respiratory health. NAT REV MICROBIOL 2017, 15:259-270.

2. Yun Y, Srinivas G, Kuenzel S, Linnenbrink M, Alnahas S, Bruce KD, Steinhoff U, Baines JF, Schaible UE: Environmentally Determined Differences in the Murine Lung Microbiota and Their Relation to Alveolar Architecture. PLOS ONE 2014, 9(12):e113466.

3. Dickson RP, Erb-Downward JR, Falkowski NR, Hunter EM, Ashley SL, Huffnagle GB: The Lung Microbiota of Healthy Mice Are Highly Variable, Cluster by Environment, and Reflect Variation in Baseline Lung Innate Immunity. AM J RESP CRIT CARE 2018, 198(4):497-508.

4. Pattaroni C, Watzenboeck ML, Schneidegger S, Kieser S, Wong NC, Bernasconi E, Pernot J, Mercier L, Knapp S, Nicod LP et al: Early-Life Formation of the Microbial and Immunological Environment of the Human Airways. CELL HOST MICROBE 2018, 24(6):857-865.

5. Segal LN, Clemente JC, Tsay JJ, Koralov SB, Keller BC, Wu BG, Li Y, Shen N, Ghedin E, Morris A et al: Enrichment of the lung microbiome with oral taxa is associated with lung inflammation of a Th17 phenotype. NAT MICROBIOL 2016, 1(5).

6. Bosch AATM, de Steenhuijsen Piters WAA, van Houten MA, Chu MLJN, Biesbroek G, Kool J, Pernet P, de Groot PCM, Eijkemans MJC, Keijser BJF et al: Maturation of the Infant Respiratory Microbiota, Environmental Drivers, and Health Consequences. A Prospective Cohort Study. AM J RESP CRIT CARE 2017, 196(12):1582-1590.

7. Adar SD, Huffnagle GB, Curtis JL: The respiratory microbiome: an underappreciated player in the human response to inhaled pollutants? ANN EPIDEMIOL 2016, 26(5):355-359. 
8. Wang Z, Bafadhel M, Haldar K, Spivak A, Mayhew D, Miller BE, Tal-Singer R, Johnston SL, Ramsheh MY, Barer MR et al: Lung microbiome dynamics in COPD exacerbations. EUR RESPIR J 2016, 47(4):1082-1092.

9. O Dwyer DN, Ashley SL, Gurczynski SJ, Xia M, Wilke C, Falkowski NR, Norman KC, Arnold KB, Huffnagle GB, Salisbury ML et al: Lung Microbiota Contribute to Pulmonary Inflammation and Disease Progression in Pulmonary Fibrosis. AM J RESP CRIT CARE 2019, 199(9):1127-1138.

10. Mayer-Hamblett N, Retsch-Bogart G, Kloster M, Accurso F, Rosenfeld M, Albers G, Black P, Brown P, Cairns A, Davis SD et al: Azithromycin for Early Pseudomonas Infection in Cystic Fibrosis. The OPTIMIZE Randomized Trial. Am J Respir Crit Care Med 2018, 198:1177-1187.

11. Chalmers JD, Boersma W, Lonergan M, Jayaram L, Crichton ML, Karalus N, Taylor SL, Martin ML, Burr LD, Wong $\mathrm{C}$ et al: Long-term macrolide antibiotics for the treatment of bronchiectasis in adults: an individual participant data meta-analysis. Lancet Respir Med 2019, 7:845-854.

12. Vermeersch K, Gabrovska M, Aumann J, Demedts IK, Corhay J, Marchand E, Slabbynck H, Haenebalcke C, Haerens M, Hanon S et al: Azithromycin during Acute COPD Exacerbations Requiring Hospitalization (BACE): a Multicentre, Randomized, Double-blind, Placebo-controlled Trial. AM J RESP CRIT CARE 2019, 200:857-868.

13. Gibson PG, Yang IA, Upham JW, Reynolds PN, Hodge S, James AL, Jenkins C, Peters MJ, Marks GB, Baraket $\mathrm{M}$ et al: Effect of azithromycin on asthma exacerbations and quality of life in adults with persistent uncontrolled asthma (AMAZES): a randomised, double-blind, placebo-controlled trial. LANCET 2017, 390:659-668.

14. Welte T: Azithromycin, the Holy Grail to Prevent Exacerbations in Chronic Respiratory Disease. AM J RESP CRIT CARE 2019, 200:269-270.

15. Taylor SL, Leong LEX, Mobegi FM, Choo JM, Wesselingh S, Yang IA, Upham JW, Reynolds PN, Hodge $\mathrm{S}$, James AL et al: Long-Term Azithromycin Reduces Haemophilus influenzae and Increases Antibiotic Resistance in Severe Asthma. AM J RESP CRIT CARE 2019, 200:309-317.

16. Rogers GB, Bruce KD, Martin ML, Burr LD, Serisier DJ: The effect of long-term macrolide treatment on respiratory microbiota composition in non-cystic fibrosis bronchiectasis: an analysis from the randomised, double-blind, placebo-controlled BLESS trial. Lancet Respir Med 2014, 2:988-996.

17. Wang L, Cheng H, Wang D, Zhao B, Zhang J, Cheng L, Yao P, Di Narzo A, Shen Y, Yu J et al: Airway microbiome is associated with respiratory functions and responses to ambient particulate matter exposure. Ecotoxicol Environ Saf 2019, 167:269-277.

18. Qin T, Zhang F, Zhou H, Ren H, Du Y, Liang S, Wang F, Cheng L, Xie X, Jin A et al: High-Level PM2.5/PM10 Exposure Is Associated With Alterations in the Human Pharyngeal Microbiota Composition. FRONT MICROBIOL 2019, 10:54.

19. Davis NM, Proctor DM, Holmes SP, Relman DA, Callahan BJ: Simple statistical identification and removal of contaminant sequences in marker-gene and metagenomics data. MICROBIOME 2018, $6(1): 226$. 
20. Moossavi S, Engen PA, Ghanbari R, Green SJ, Naqib A, Bishehsari F, Merat S, Poustchi H, Keshavarzian A, Malekzadeh R: Assessment of the impact of different fecal storage protocols on the microbiota diversity and composition: a pilot study. BMC MICROBIOL 2019, 19(1).

21. Parnham MJ, Erakovic HV, Giamarellos-Bourboulis EJ, Perletti G, Verleden GM, Vos R: Azithromycin: mechanisms of action and their relevance for clinical applications. Pharmacol Ther 2014, 143(2):225-245.

22. Wierzbowski AK, Karlowsky JA, Adam HJ, Nichol KA, Hoban DJ, Zhanel GG: Evolution and molecular characterization of macrolide-resistant Streptococcus pneumoniae in Canada between 1998 and 2008. J Antimicrob Chemother 2013, 69(1):59-66.

23. Huang CY, Lai JF, Huang IW, Chen PC, Wang HY, Shiau YR, Cheng YW, Hsieh LY, Chang SC, Lauderdale TL: Epidemiology and molecular characterization of macrolide-resistant Streptococcus pyogenes in Taiwan. J CLIN MICROBIOL 2014, 52(2):508-516.

24. Yayan J: The comparative development of elevated resistance to macrolides in community-acquired pneumonia caused by Streptococcus pneumoniae. Drug Des Devel Ther. 2014, 8:1733-1743.

25. Hu F, Zhu D, Wang F, Wang M: Current Status and Trends of Antibacterial Resistance in China. CLIN INFECT DIS 2018, 67(suppl_2):S128-S134.

26. Zhao C, Zhang F, Wang Z, Cao B, Xu X, Sun H, Zhang R, Hu Y, Liu Z, Du Y et al: Resistance surveillance of major pathogens for adult community-acquired respiratory tract infections in China: a multicenter study 2012. Zhong hua Jie He He Hu Xi Za Zhi 2015, 1(38):18-22.

27. Palleja A, Mikkelsen KH, Forslund SK, Kashani A, Allin KH, Nielsen T, Hansen TH, Liang S, Feng Q, Zhang $\mathrm{C}$ et al: Recovery of gut microbiota of healthy adults following antibiotic exposure. NAT MICROBIOL 2018, 3(11):1255-1265.

28. Liu C, Chen R, Sera F, Vicedo-Cabrera AM, Guo Y, Tong S, Coelho MSZS, Saldiva PHN, Lavigne E, Matus P et al: Ambient Particulate Air Pollution and Daily Mortality in 652 Cities. N Engl J Med 2019, 381:705-715.

29. Rice MB, Ljungman PL, Wilker EH, Dorans KS, Gold DR, Schwartz J, Koutrakis P, Washko GR, O'Connor GT, Mittleman MA: Long-term exposure to traffic emissions and fine particulate matter and lung function decline in the Framingham heart study. Am J Respir Crit Care Med 2015, 191(6):656664.

30. Brunst KJ, Ryan PH, Brokamp C, Bernstein D, Reponen T, Lockey J, Khurana HG, Levin L, Grinshpun SA, LeMasters G: Timing and Duration of Traffic-related Air Pollution Exposure and the Risk for Childhood Wheeze and Asthma. Am J Respir Crit Care Med 2015, 192(4):421-427.

31. Jacquemin B, Siroux V, Sanchez M, Carsin AE, Schikowski T, Adam M, Bellisario V, Buschka A, Bono $\mathrm{R}$, Brunekreef $\mathrm{B}$ et al: Ambient air pollution and adult asthma incidence in six European cohorts (ESCAPE). Environ Health Perspect 2015, 123(6):613-621.

32. Dominici F, Peng RD, Bell ML, Pham L, McDermott A, Zeger SL, Samet JM: Fine particulate air pollution and hospital admission for cardiovascular and respiratory diseases. JAMA 2006, 295(10):1127-1134. 
33. Hamra GB, Guha N, Cohen A, Laden F, Raaschou-Nielsen O, Samet JM, Vineis P, Forastiere F, Saldiva $\mathrm{P}$, Yorifuji $\mathrm{T}$ et al: Outdoor particulate matter exposure and lung cancer: a systematic review and meta-analysis. Environ Health Perspect 2014, 122(9):906-911.

34. Reilly JP, Zhao Z, Shashaty MGS, Koyama T, Christie JD, Lanken PN, Wang C, Balmes JR, Matthay MA, Calfee CS et al: Low to Moderate Air Pollutant Exposure and Acute Respiratory Distress Syndrome after Severe Trauma. AM J RESP CRIT CARE 2019, 199(1):62-70.

35. Alderete TL, Jones RB, Chen Z, Kim JS, Habre R, Lurmann F, Gilliland FD, Goran MI: Exposure to traffic-related air pollution and the composition of the gut microbiota in overweight and obese adolescents. ENVIRON RES 2018, 161:472-478.

36. Zhong S, Zhang L, Jiang X, Gao P: Comparison of chemical composition and airborne bacterial community structure in PM2.5 during haze and non-haze days in the winter in Guilin, China. SCl TOTAL ENVIRON2019, 655:202-210.

37. Sommer F, Anderson JM, Bharti R, Raes J, Rosenstiel P: The resilience of the intestinal microbiota influences health and disease. NAT REV MICROBIOL 2017, 15(10):630-638.

38. Moya A, Ferrer M: Functional Redundancy-Induced Stability of Gut Microbiota Subjected to Disturbance. TRENDS MICROBIOL 2016, 24(5):402-413.

39. O'Beirne SL, Shenoy SA, Salit J, Strulovici-Barel Y, Kaner RJ, Visvanathan S, Fine JS, Mezey JG, Crystal RG: Ambient Pollution-related Reprogramming of the Human Small Airway Epithelial Transcriptome. Am J Respir Crit Care Med 2018, 198(11):1413-1422.

40. Brown RL, Sequeira RP, Clarke TB: The microbiota protects against respiratory infection via GM-CSF signaling. NAT COMMUN2017, 8(1):1512.

41. Feng K, Zhang Z, Cai W, Liu W, Xu M, Yin H, Wang A, He Z, Deng Y: Biodiversity and species competition regulate the resilience of microbial biofilm community. MOL ECOL 2017, 26:6170-6182.

42. Tao J, Meng D, Qin C, Liu X, Liang Y, Xiao Y, Liu Z, Gu Y, Li J, Yin H: Integrated network analysis reveals the importance of microbial interactions for maize growth. App/ Microbiol Biotechnol 2018, 102(8):3805-3818.

43. Montesinos-Navarro A, Hiraldo F, Tella JL, Blanco G: Network structure embracing mutualismantagonism continuums increases community robustness. NAT ECOL EVOL 2017, 1(11):1661-1669.

44. Ruiz VE, Battaglia T, Kurtz ZD, Bijnens L, Ou A, Engstrand I, Zheng X, lizumi T, Mullins BJ, Müller CL et al: A single early-in-life macrolide course has lasting effects on murine microbial network topology and immunity. NAT COMMUN2017, 8(1).

45. Zaura E, Brandt BW, Teixeira De Mattos MJ, Buijs MJ, Caspers MPM, Rashid MU, Weintraub A, Nord $\mathrm{CE}$, Savell A, Hu Y et al: Same exposure but two radically different responses to antibiotics: resilience of the salivary microbiome versus long-term microbial shifts in feces. MBIO 2015, 6(6):e1693.

46. Choo JM, Abell G, Thomson R, Morgan L, Waterer G, Gordon DL, Taylor SL, Leong L, Wesselingh SL, Burr LD et al: Impact of Long-Term Erythromycin Therapy on the Oropharyngeal Microbiome and Resistance Gene Reservoir in Non-Cystic Fibrosis Bronchiectasis. MSPHERE 2018, 3(2). 
47. Available from: https://support.illumina.com/content/dam/illuminasupport/documents/documentation/chemistry_documentation/16s/16s-metagenomic-library-prepguide-15044223-b.pdf..

48. Rognes T, Flouri T, Nichols B, Quince C, Mahe F: VSEARCH: a versatile open source tool for metagenomics. PEERJ 2016, 4:e2584.

49. Edgar RC: UPARSE: highly accurate OTU sequences from microbial amplicon reads. NAT METHODS 2013, 10(10):996-998.

50. Edgar RC: UNOISE2: Improved Error-Correction for Illumina 16S and ITS Amplicon Sequencing. bioRxiv 2016.

51. Edgar RC: SINTAX: a simple non-Bayesian taxonomy classifier for $16 \mathrm{~S}$ and ITS sequences. bioRxiv 2016, 1:1-10.

52. Gu Z: Complex heatmaps reveal patterns and correlations in multidimensional genomic data. BIOINFORMATICS 2016.

\section{Figures}

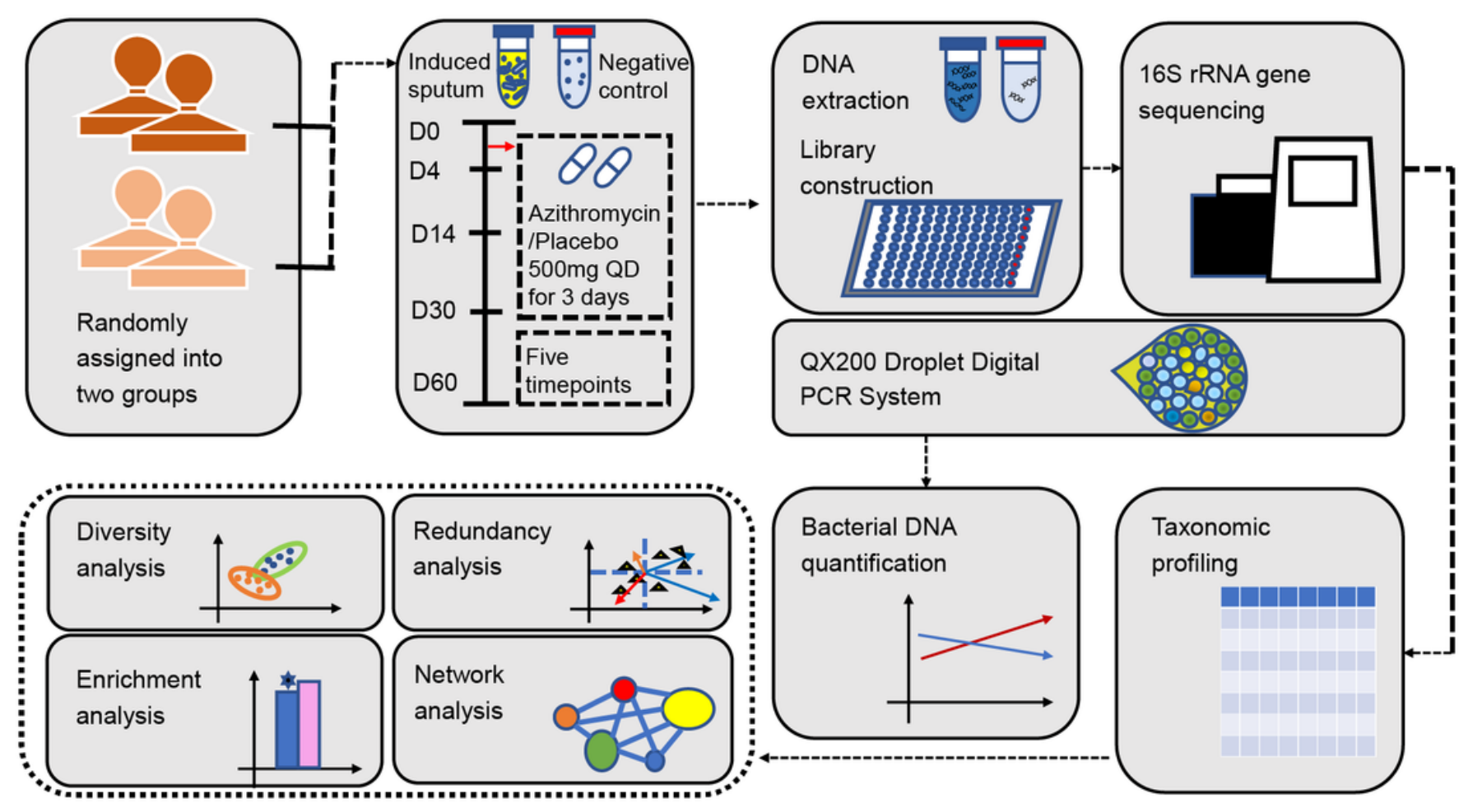

\section{Figure 1}

Overview of the study design and sample collection. The healthy volunteers $(n=48)$ enrolled in this study are stratified by gender and randomly assigned to either the azithromycin or the placebo group (1:1). $500 \mathrm{mg}$ azithromycin or $500 \mathrm{mg}$ starch tablets which are contained in an identical opaque white capsule is 
administered once daily for 3 days. The sputum samples are collected at the day before the drugs administration (D0), the day after the treatment course is completed (D4), 14 days, 30 days and 60 days post-dosing. 16S rRNA gene sequencing is applied to the induced sputum samples and negative control samples. Bacterial DNA of sputum samples is quantified using a QX200 Droplet Digital PCR System. The microbial ecology analysis is performed on the data produced from the study later.

A

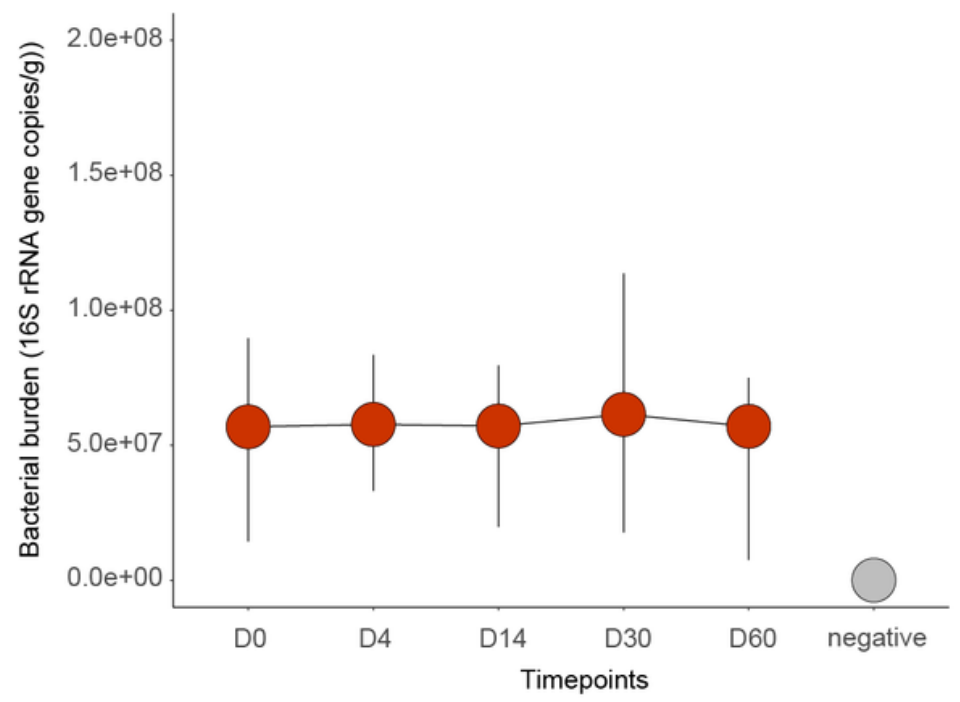

B

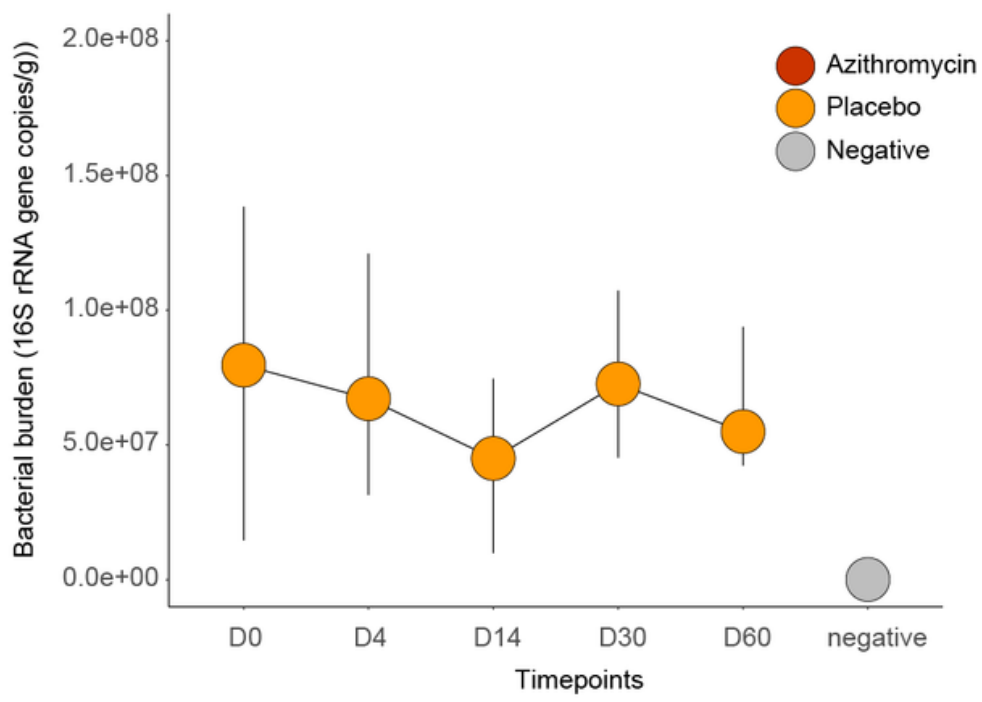

Figure 2

Sputum bacterial DNA quantification using droplet digital PCR of the 16S rRNA gene. There is no significant difference in sputum bacterial DNA burden across different timepoints in either azithromycin group (A) or placebo group (B) (Wilcoxon signed-rank test, Bonferroni adjusted $P$ values $(q)>0.05$ for all). 

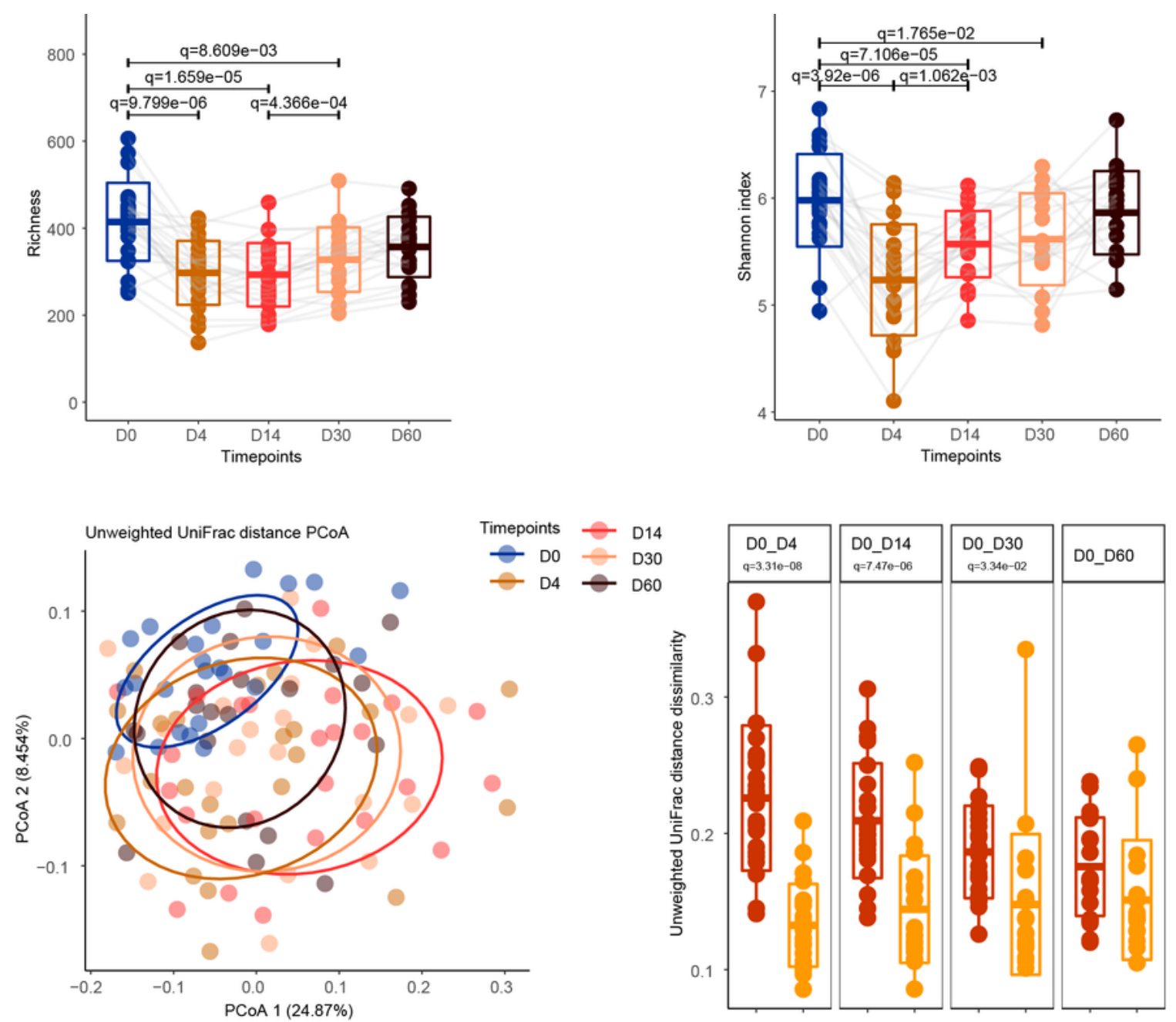

Figure 3

Airway microbial diversity and community composition changes in azithromycin group. (A) Species richness. (B) Shannon index. The boxplots represent the diversity measures for the subjects (centre line, mean; box limits, \pm standard deviation; whisker limits, maximum/minimum). The points are connected across timepoints by grey lines. PCoA plot based on unweighted UniFrac distance (C) and weighted UniFrac distance (E). The ellipses represent the $68 \%$ confidence interval for each timepoint. The boxplots 
show the unweighted UniFrac distance (D) or weighted UniFrac distance (F) dissimilarities between samesubject samples collecting from two different timepoints (centre line, mean; box limits, \pm standard deviation; whisker limits, maximum/minimum).

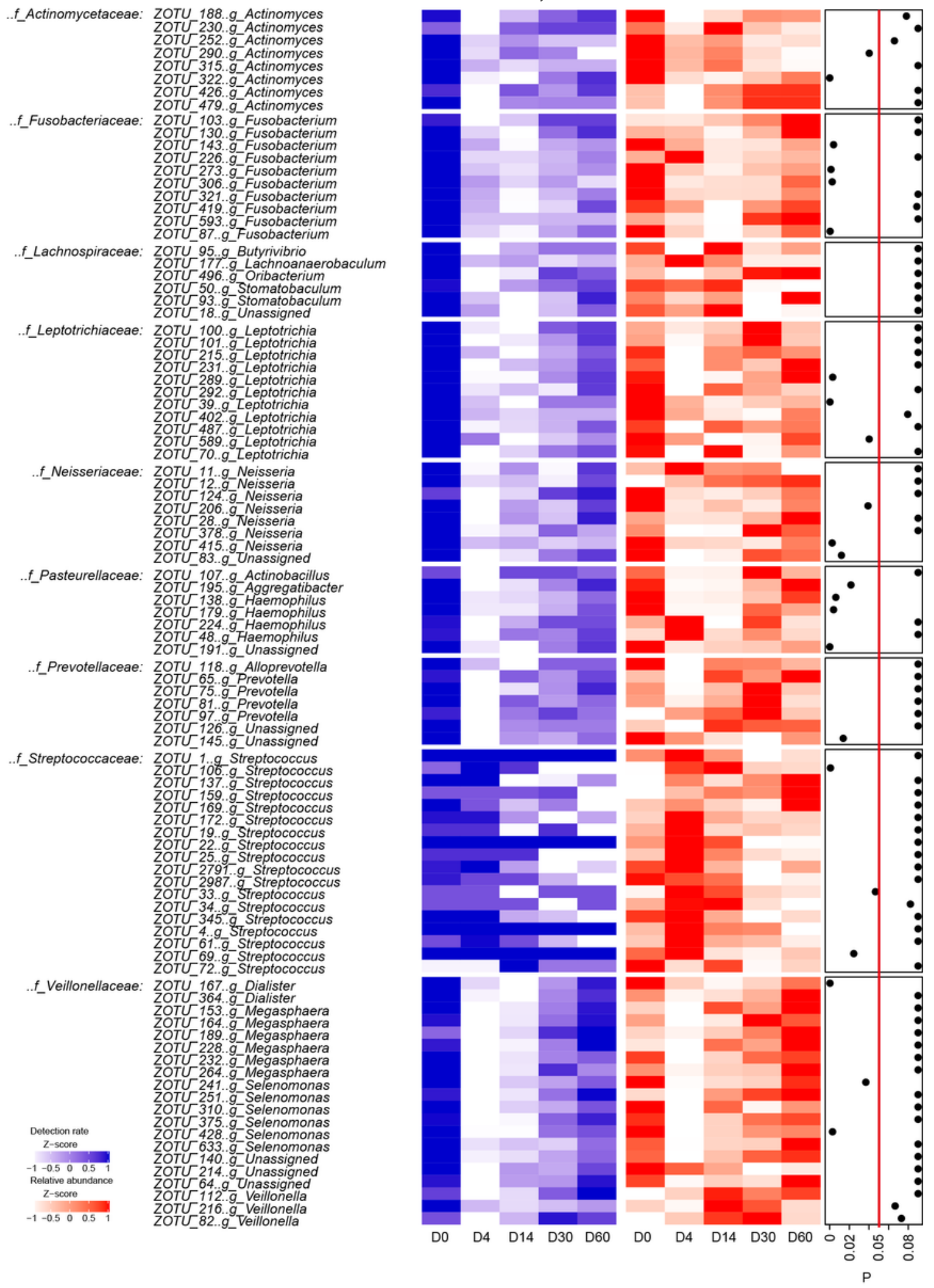

Figure 4

Microbial taxonomic variation after azithromycin administration during 60 days' follow-up. * $\mathrm{P}$ value $\geq 0.05$ : there isn't a significant difference in the relative abundance of the species at D14 compared 
to $D 0 ; P$ value 0.05 : there is a significant difference in the relative abundance of the species at D14 compared to D0.

A

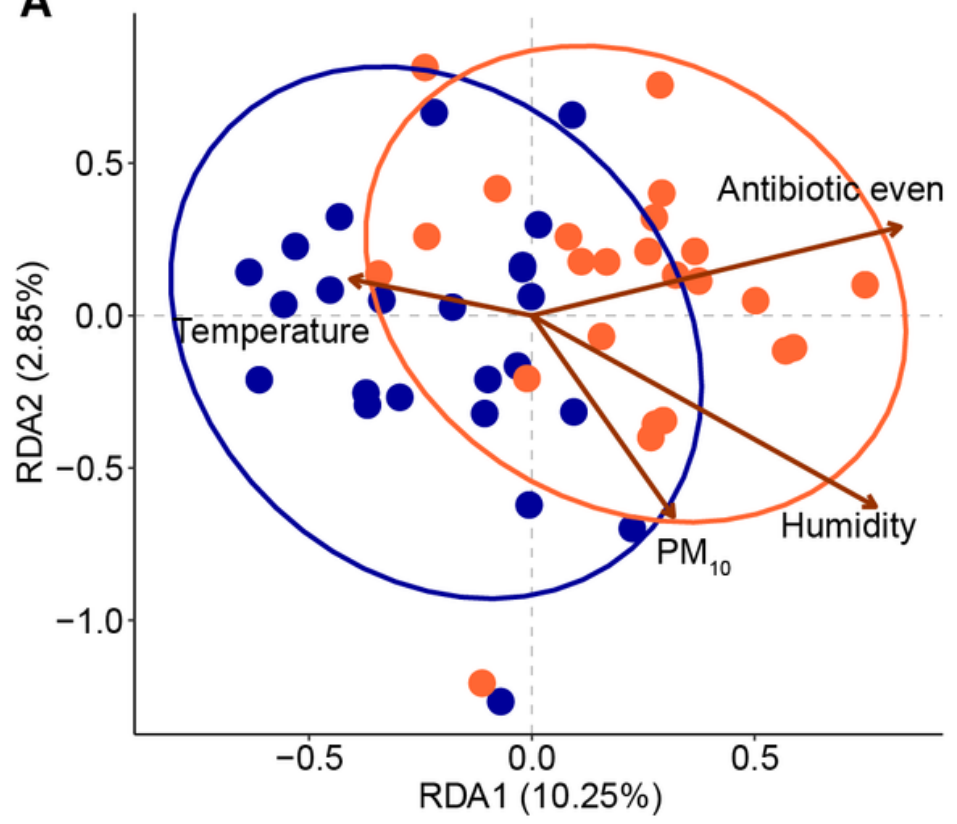

B

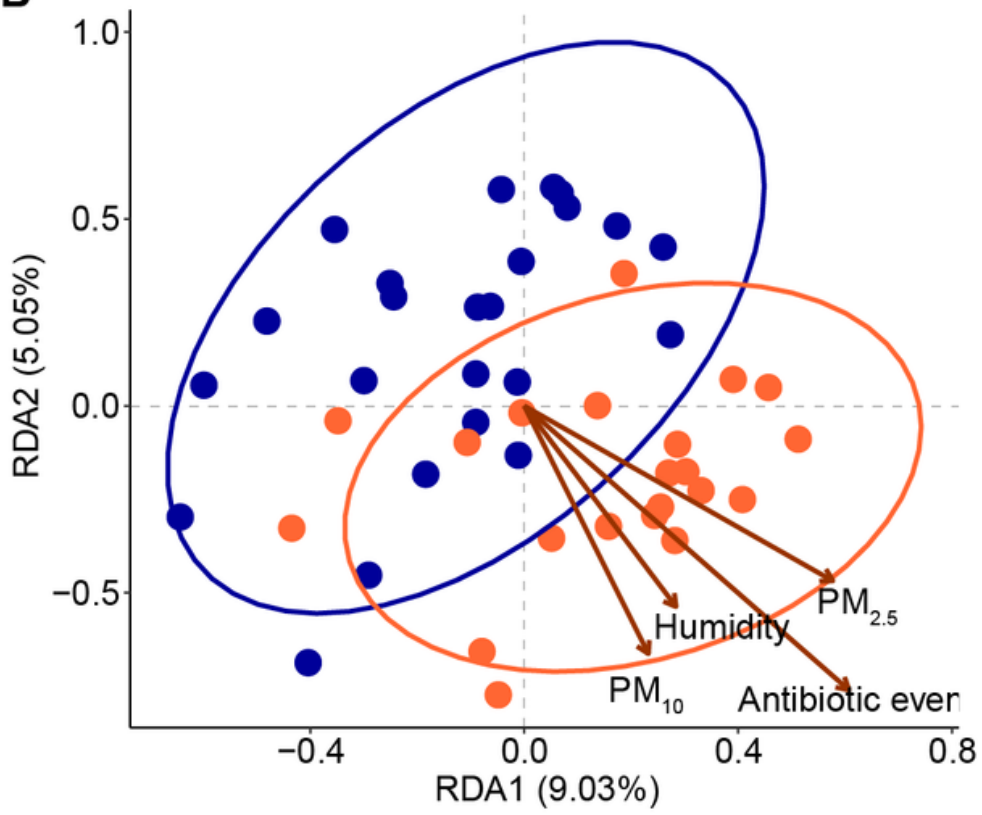

Antibiotic-before

Antibiotic-after

Figure 5

Distance-based redundancy analysis (db-RDA) based on weighted UniFrac distance. (A) The relation of the environmental factors, antibiotic event and the sputum microbiota variation at D4. (B) The relation of the environmental factors, antibiotic event and the sputum microbiota variation at D30. The ellipses represent the $95 \%$ confidence interval for each timepoint. 


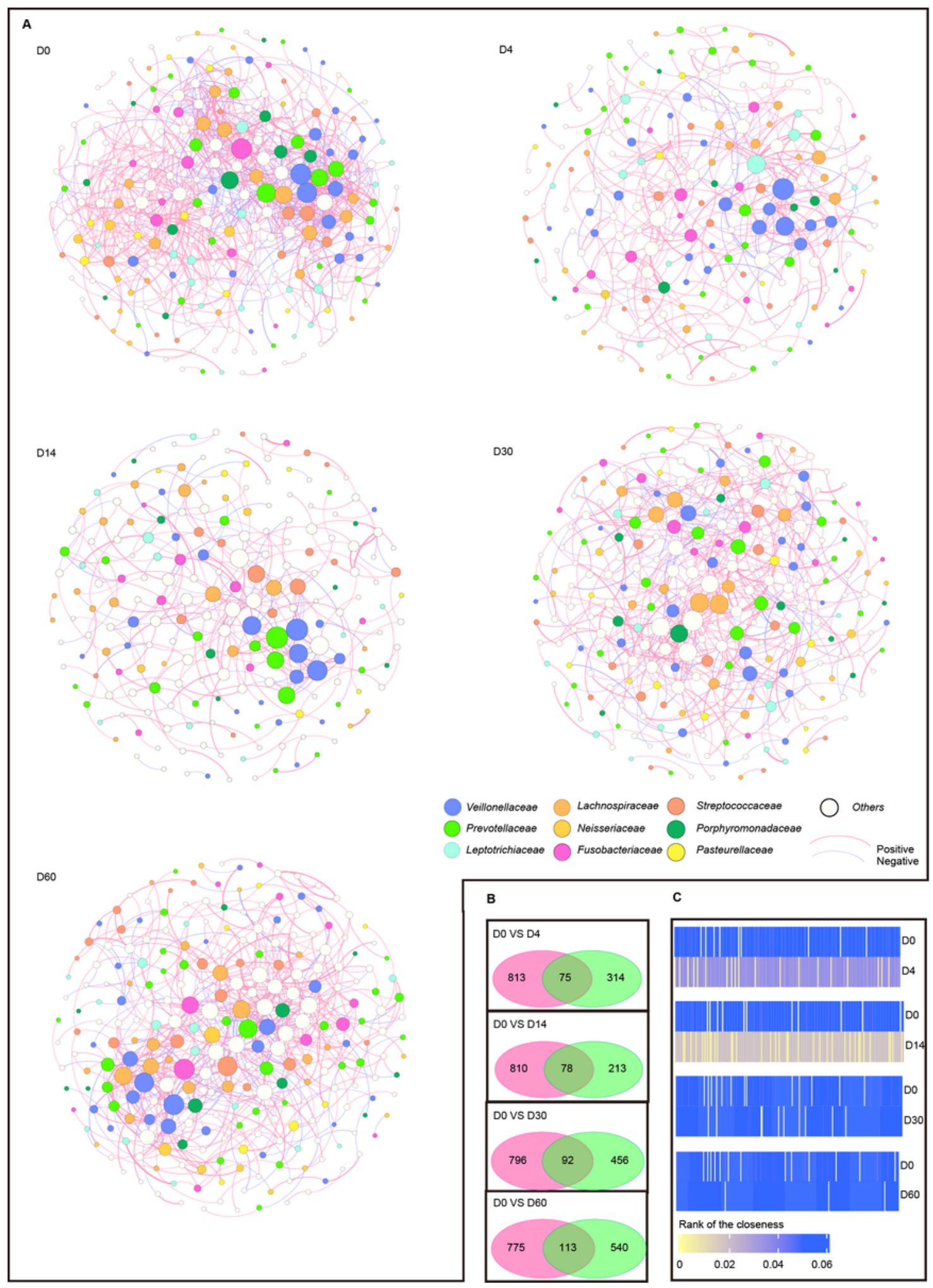

\section{Figure 6}

The network analysis in azithromycin group. (A) Networks of co-occurring ZOTUs in airway microbiota for timepoints D0, D4, D14, D30 and D60. Nodes are colored by ZOTU families, with size proportional to mean relative abundance, and edge width proportional to confidence score. (B) The number of shared edges between network D0 and timepoints D4, D14, D30, D60. (C) The closeness centralization of shared nodes between network D0 and timepoints D4, D14, D30, D60. 


\section{Supplementary Files}

This is a list of supplementary files associated with this preprint. Click to download.

- TableS4.docx

- TableS3.xIsx

- TableS2.xlsx

- TableS1.xlsx

- FigureS17.tif

- FigureS16.tif

- FigureS15.tif

- FigureS14.tif

- FigureS13.tif

- FigureS12.tif

- FigureS11.tif

- FigureS10.tif

- Figures9.tif

- Figures8.tif

- FigureS7.tif

- FigureS6.tif

- FigureS5.tif

- FigureS4.tif

- FigureS3.tif

- Figures2.tif

- FigureS1.tif

- Supplement.docx 